\title{
Towards an effective field theory for vector mesons
}

\author{
Stefan Leupold* and Carla Terschlüsen \\ Department of Physics and Astronomy, Uppsala University, Sweden \\ E-mail: stefan.leupoldephysics.uu.se
}

\begin{abstract}
The assumption that vector mesons dominate the interactions of hadrons with electromagnetism (vector-meson dominance - VMD) provides an important phenomenological concept. On the other hand, a clear microscopic derivation is still missing and there are cases where VMD drastically fails, e.g. for the omega transition form factor. In principle, effective field theories with their systematic expansion and power counting could provide a tool to assess the validity of VMD and more generally to describe the interactions of vector mesons at low energies. Though the systematic development is still in an infant stage we present here a Lagrangian for light pseudoscalar and vector mesons which is inspired by ideas from effective field theories. The Lagrangian is used to calculate electromagnetic meson form factors. It turns out that one can reproduce both the successes of VMD concerning the pion form factors and the deviations from VMD concerning the omega transition form factor.
\end{abstract}

50th International Winter Meeting on Nuclear Physics

23-27 January 2012

Bormio, Italy

\footnotetext{
*Speaker.
} 


\section{Introduction}

A good understanding of the interactions of hadrons with electromagnetism is a key ingredient for several areas of hadron and particle physics. To name a few examples: a) The first indication that the proton has an intrinsic structure came from elastic electron-proton scattering [1]. Followup experiments for elastic and (deep) inelastic scattering revealed more and more of the proton structure, nowadays quantitatively encoded in structure functions, electromagnetic form factors, generalized parton distributions and so on. b) The change of the properties of hadrons once they are placed in a strongly interacting environment is an active field of research in heavy-ion and neutronstar physics. Such in-medium properties of hadrons can be accessed in dilepton spectra [2,3]. c) A promising candidate to search for physics beyond the standard model is the gyromagnetic ratio of the muon. At present the largest uncertainty on the theory side for the standard-model calculation is provided by the hadronic contribution to this gyromagnetic ratio [4].

So, what do we know about the interactions between hadrons and real or virtual photons? The neutral vector mesons which have the same quantum numbers as the photon can couple directly to the photon. Indeed, these vector mesons are prominently seen in the corresponding cross sections and decay rates. This gave rise to the notion of vector-meson dominance (VMD) [5]. In the following, we will concentrate on electromagnetic meson form factors. We start with a brief review how well such form factors are described by VMD.

The pion form factor is determined by the reaction $e^{+} e^{-} \rightarrow \pi^{+} \pi^{-}$. It constitutes one of the traditional successes of the VMD scenario [5]. Figure 1 shows how well VMD works for this form factor. In contrast, VMD drastically fails for the omega transition form factor, extracted from

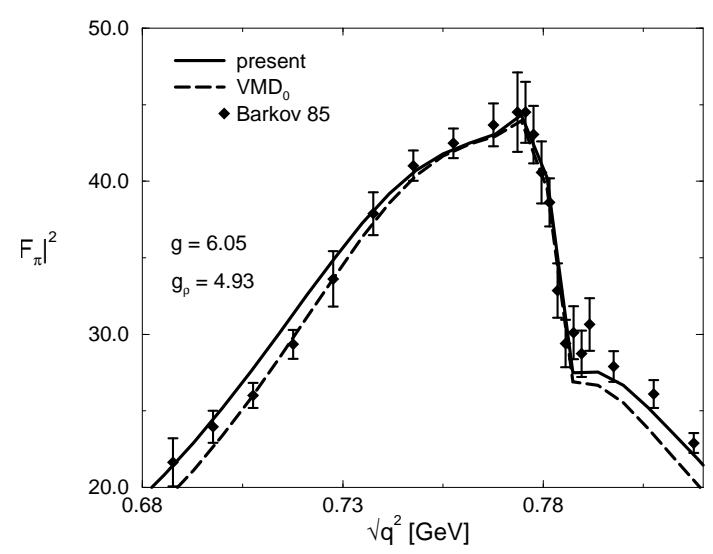

Figure 1: The pion form factor as compared to VMD. Figure taken from [6].

the reaction $\omega \rightarrow \mu^{+} \mu^{-} \pi^{0}$. This is displayed one the left-hand side of figure 2 where the VMD prediction is compared to data from NA60 and Lepton G [7, 8]. For the eta transition form factor, extracted from the reaction $\eta \rightarrow \mu^{+} \mu^{-} \gamma$, VMD works very well as can be seen on the right-hand side of figure 2. Also for the pion transition form factor, extracted, e.g., from $e^{-} \gamma \rightarrow e^{-} \pi^{0}$, the VMD scenario works well. Here, a key quantity is the slope of the form factor at vanishing mass 

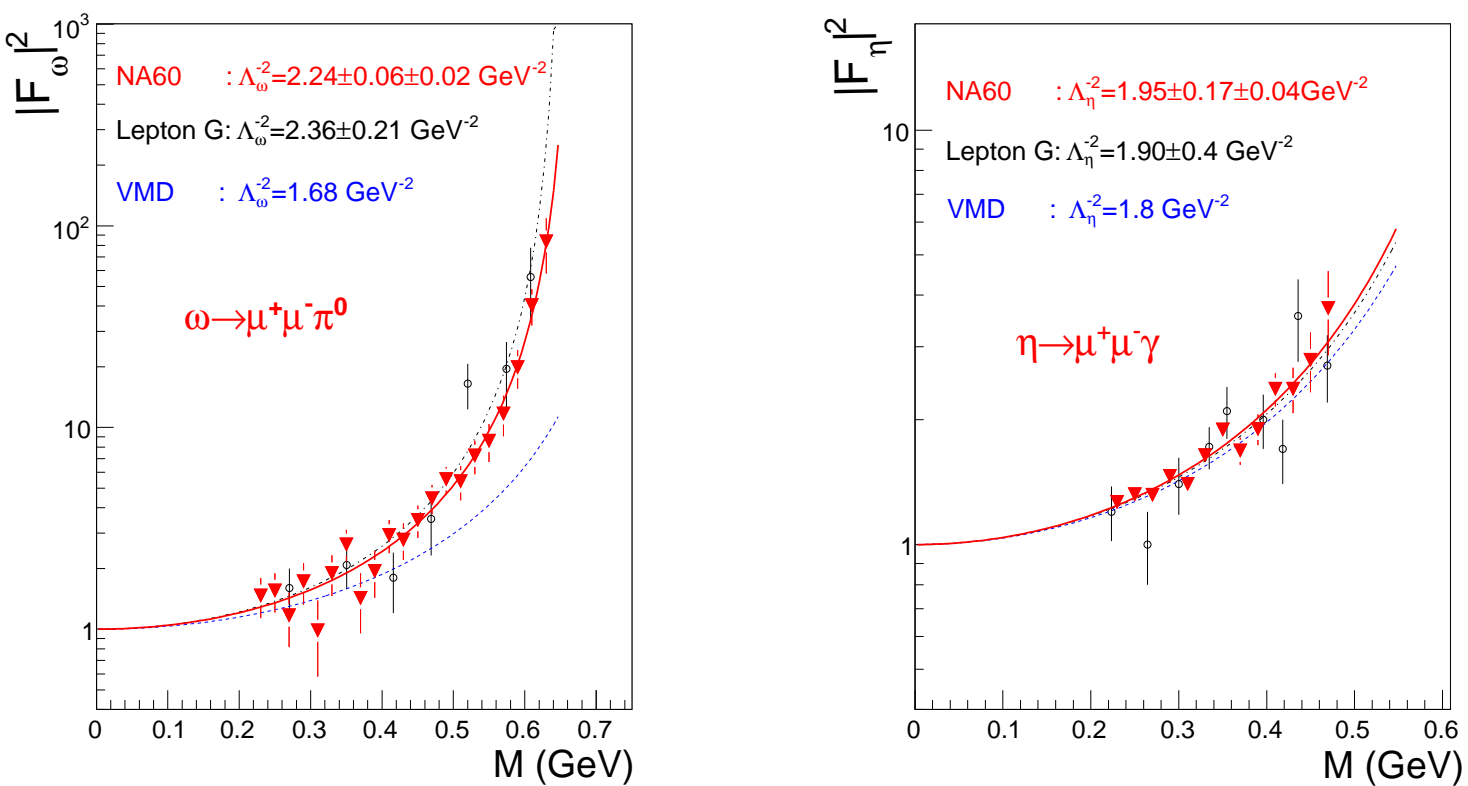

Figure 2: The transition form factors of omega to pion (left) and of eta to photon (right) as compared to VMD. The only theory curves are the dashed VMD lines. The full and dash-dotted lines are fits to the data. Figures taken from [8].

of the virtual photon,

$$
\Lambda^{-2}:=\left.\frac{d F(s)}{d s}\right|_{s=0}
$$

The VMD prediction is ( $m_{V}$ denotes the $\rho$-meson mass)

$$
\Lambda_{\mathrm{VMD}}=m_{V} \approx 0.77 \mathrm{GeV}
$$

which is in excellent agreement with the experimental result [9]

$$
\Lambda_{\mathrm{PDG}}=(0.76 \pm 0.05) \mathrm{GeV}
$$

This quantity concerns the single-virtual pion transition form factor, i.e. the electromagnetic transition between a pion and a real photon. Also the double-virtual pion transition form factor is of considerable interest. For example, it provides an important part of the hadronic light-by-light scattering contribution to the magnetic moment of the muon [4]. Therefore, it is important to clarify whether the double-virtual pion transition form factor can be reliably described by VMD (see, e.g., $[10,11,12])$. This issue has not been settled yet from the experimental side. In principle, reactions like $\pi^{0} \rightarrow 2 e^{+} 2 e^{-}$and $e^{-} e^{+} \rightarrow e^{-} e^{+} \pi^{0}$ have to be studied. We will come back to this issue below.

\section{Effective field theories}

In the following we will describe first steps towards an effective field theory for vector mesons. One aim of such an enterprise is the reliable calculation of electromagnetic form factors and other 
quantities where vector mesons constitute important degrees of freedom. In that way it should be possible to figure out where or under which conditions VMD works and where it does not. It should be stressed right away that at present this development is in an infant stage. Though first results are encouraging - as will be shown below - it has not been established yet that a systematic low-energy expansion is feasible and convergent for the energy region where vector mesons are important. Below, a Lagrangian for light vector and pseudoscalar mesons will be presented which is inspired by the ideas of effective field theories. Without an established systematic power counting this Lagrangian might still be regarded at present as a - rather successful - phenomenological tree-level model. However, the ambition of this enterprise is higher and one-loop calculations are under way to scrutinize the validity of this approach as a systematic effective field theory.

The basic idea of an effective field theory is to perform a Taylor expansion in the involved momenta. (For this general outline we do not specify whether we talk about four- or three-momenta - see the more detailed discussion below.) This is a reasonable approach as long as one sticks to processes with low enough momenta. The crucial question is what "low enough" means quantitatively. We will come back to this question below. The difference between an effective field theory and a phenomenological model (like, e.g., VMD) is that for the construction of the Lagrangian one considers all possible terms which are in agreement with the symmetries of the system. ${ }^{1}$ Then, the basic idea is to classify these possible terms according to their number of derivatives. The reason simply is that derivatives translate to momenta. Thus, the more derivatives a term in the Lagrangian has, the less important it is for low-energy processes. The generic feature of an effective field theory is that one can systematically improve a calculation by working out the next order in the Taylor expansion. In that sense it is like an ordinary perturbative field theory. The difference is just that one does not expand in powers of the coupling constants but in powers of the involved (small!) momenta. Typically, higher powers in the Taylor expansion involve higher-loop calculations. In contrast, in a phenomenological model one demands from outside to restrict the calculations, e.g., to tree level or to one-loop level without a reason why the neglected contributions should be small. An effective field theory is defined by the most general Lagrangian one can write down together with a power counting (and the quantization procedure). All emerging Feynman diagrams can be classified according to the power counting. Therefore, one knows at which order in the Taylor expansion which diagrams contribute. In contrast, a phenomenological model is defined by a specific Lagrangian together with a "by-hand" prescription which Feynman diagrams should be considered. In that sense an effective field theory is systematic while a phenomenological model is not. On the other hand, one cannot always formulate an effective field theory for a given energy region. The point is that an effective field theory is only feasible, i.e. effective, if the Taylor expansion in the involved momenta converges (fast enough). Therefore the quantitatively important question is: What is the dimensionless quantity the Taylor expansion (power counting) is based on? In the numerator one has the momenta, the so-called "soft scale". But which quantity appears in the denominator? This "hard scale" needs to be significantly larger than the considered momentum region. Only then the effective field theory can work. This discussion will be continued below when we have specified the respective Lagrangians.

\footnotetext{
${ }^{1}$ For the strong interaction in the sector of hadrons made out of light quarks this includes parity, charge conjugation and approximate chiral symmetry.
} 
In the following we restrict ourselves to mesons built out of the lightest two quark flavors, namely the pseudoscalar pions, encoded in the flavor matrix

$$
\Phi=\left(\begin{array}{cc}
\pi^{0} & \sqrt{2} \pi^{+} \\
\sqrt{2} \pi^{-} & -\pi^{0}
\end{array}\right),
$$

and the rho and omega vector mesons, collected in

$$
V_{\mu \nu}=\left(\begin{array}{cc}
\rho^{0}+\omega & \sqrt{2} \rho^{+} \\
\sqrt{2} \rho^{-} & -\rho^{0}+\omega
\end{array}\right)_{\mu \nu} .
$$

Strictly speaking, most of the results which will be presented below have been obtained in a framework $[13,14,15,12]$ which included three active quark flavors - up, down and strange. There, the octet of pseudoscalar mesons ( $\pi$ 's, $K$ 's, $\eta$ ) together with the nonet of vector mesons ( $\rho$ 's, $K^{*}$ 's, $\omega$, $\varphi$ ) has been taken into account. However, a fully consistent framework had to include the full flavor nonets of pseudoscalar and vector mesons. This implies that one needs to incorporate also the flavor singlet $\eta$ and its mixing with the octet $\eta$ to the physical states $\eta$ and $\eta^{\prime}$. Such an extension is presently under construction [16].

For the energy region where the light pseudoscalar mesons are the only relevant degrees of freedom the appropriate effective field theory is well established; it is called "chiral perturbation theory" $(\chi \mathrm{PT})[17,18,19,20]$. We shall briefly review the respective leading-order chiral Lagrangian for the sector with an even and with an odd number of pseudoscalar mesons, respectively. Afterwards we shall discuss the inclusion of vector mesons.

In the sector of natural parity - where one only has terms with an even number of pseudoscalar mesons and where on the formal level no Levi-Civita tensor appears - the part of the leading-order $\chi$ PT Lagrangian relevant for the present purpose is given by [17, 18, 19, 20]

$$
\mathscr{L}_{\chi \mathrm{PT}}=f^{2} \operatorname{tr}\left(U_{\mu}^{\dagger} U^{\mu}\right)+\frac{1}{4} f^{2} m_{\pi}^{2} \operatorname{tr}\left(U^{\dagger}+U\right) .
$$

Here, "tr" is the flavor trace, $f \approx 90 \mathrm{MeV}$ denotes the pion decay constant and $m_{\pi}$ the pion mass. The pion fields are encoded in

$$
U=u^{2}=\exp (\mathrm{i} \Phi / f), \quad U_{\mu}=\frac{1}{2} u^{\dagger}\left(D_{\mu} U\right) u^{\dagger}
$$

with the gauge covariant derivative

$$
D_{\mu} U=\partial_{\mu} U+i e[Q, U] A_{\mu},
$$

the positron charge $e$, the quark charge matrix

$$
Q=\left(\begin{array}{cc}
+\frac{2}{3} & 0 \\
0 & -\frac{1}{3}
\end{array}\right)
$$

and the photon field $A_{\mu}$. We have neglected isospin breaking and external fields other than the electromagnetic one. The non-linear appearance of the pion fields (2.1) in (2.4) is caused by the spontaneous breaking of chiral symmetry. One implication is that already the chirally extended 
kinetic term, $f^{2} \operatorname{tr}\left(U_{\mu}^{\dagger} U^{\mu}\right)$, contains interactions with a fixed interaction strength given by the pion decay constant. For example, the corresponding four-point interaction is $\sim \frac{1}{f^{2}}\left[\Phi, \partial_{\mu} \Phi\right]\left[\Phi, \partial^{\mu} \Phi\right]$.

For reactions of pions with pions and/or photons the tree-level diagrams emerging from the leading-order Lagrangian provide a decent description - as long as the reaction energy is small enough. ${ }^{2}$ If one assigns a generic momentum $p$ to such reactions, then all involved particle energies and three-momenta should roughly be as large as $p$ (or smaller). As a consequence also the pion mass should be of this order, i.e. $m_{\pi} \sim p$. The terms in the leading-order Lagrangian (2.3) are all of order $O\left(p^{2}\right)$ : In the first term the derivatives translate to momenta which are $\sim p$. In the second term one has the square of the pion mass. It is advantageous to count also the photon field as order $p$. In that way, both terms in the gauge covariant derivative (2.5) have formally the same order.

There can be no terms of order $O\left(p^{0}\right)$ because of the Goldstone-boson nature of the pions. There can be no terms of order $O\left(p^{1}\right)$ because of Lorentz invariance. Thus, $O\left(p^{2}\right)$ is the leading order and it is important to note that (2.3) is the most general Lagrangian of order $O\left(p^{2}\right)$ which is in agreement with the symmetries of the strong interaction, i.e., for the case at hand, parity, charge conjugation and approximate chiral symmetry. For the given setup, i.e. considering only pions as active degrees of freedom, there are no other terms of order $O\left(p^{2}\right)$. Thus, there are only two parameters ("low-energy constants") governing the lowest-order Lagrangian: the pion decay constant $f$ and the pion mass $m_{\pi}$. They can be adjusted to data or ideally deduced from the microscopic theory, QCD. Once these parameters are determined, the Lagrangian has predictive power for low-energy reactions. In addition, one can show that loop diagrams emerging from (2.3) are at least of order $O\left(p^{4}\right)$. Therefore, a tree-level calculation is sufficient at low enough energies.

If one wants to describe reactions at somewhat higher energies or the low-energy reactions with larger accuracy, then one has to work out the $O\left(p^{4}\right)$ contributions. They emerge from oneloop diagrams based on the leading-order Lagrangian (2.3) and from tree-level diagrams based on the next-to-leading-order Lagrangian. The latter contains all terms with four derivatives and/or pion masses. It involves additional low-energy constants which again needed to be fitted to additional data or deduced from QCD. In principle, one can continue this procedure to higher powers of $p$. In practice, one limitation comes from the plethora of additional parameters which come in with the higher-order Lagrangians [21, 22]. This restricts the predictive power of more accurate calculations.

However, there is an additional problem if one would like to increase the applicability range to higher energies. The convergence of the power series in $p$ is limited for, at least, two reasons. One limit emerges from the loop diagrams. The power series in $p$ breaks down if the loop diagrams become as important as the tree diagrams based on the same Lagrangian. If one works that out for chiral perturbation theory one finds that the scale is set by

$$
4 \pi f \approx 1 \mathrm{GeV} \text {. }
$$

In the following, this scale emerging from a comparison of tree-level and loop diagrams is called "intrinsic hard scale".

A second limitation comes from the fact that only the pions (or, for three flavors, the pions, kaons and eta) are taken into account. Therefore, the convergence of the series must break down in

\footnotetext{
${ }^{2}$ What "small enough" means quantitatively will be specified in a moment.
} 
the energy region where also other mesons become active degrees of freedom. For example, one might consider the $\rho$ meson. It contributes to pion-pion scattering and the corresponding Feynman diagrams contain a vector-meson propagator, $1 /\left(p^{2}-m_{V}^{2}\right)$, cf., e.g., the right-hand side of figure 5 below. Again, $p$ denotes the typical energy/momentum of the considered reaction, and $m_{V}$ denotes the vector-meson mass. For $p \ll m_{V}$ one can expand this vector-meson propagator in a power series of $p^{2} / m_{V}^{2}$. In chiral perturbation theory, where vector mesons are not considered explicitly, such contributions are encoded in the low-energy constants [23, 24]. Obviously the expansion of the vector-meson propagator breaks down if the typical momentum becomes as large as the mass of the vector meson. More generally, the masses of the not considered degrees of freedom set an energetic limit to the applicability range of an effective field theory. This range scales with the mass gap between the heaviest considered and the lightest not considered state of the effective field theory. For chiral perturbation theory it is very advantageous that the pions are much lighter than all the other mesons because of the Goldstone-boson character of the pions. The scale connected to the neglected degrees of freedom is called "external hard scale" in what follows.

In principle, it is tempting to extend chiral perturbation theory by including heavier states. In that way one could include more processes and it might be possible to push the external hard scale to higher energies. There are several aspects to consider: One has to decide whether one wants to include these additional degrees of freedom as light or as heavy as compared to the considered momentum scale $p$. For light degrees of freedom all masses, energies and momenta should be of the order of $p$. To turn the argument around, the masses and momenta define the soft scale $p$. This makes only sense if the soft scale remains significantly smaller than the intrinsic hard scale introduced above. This provides a limit for the masses of states which could be reasonably considered as "light". In addition, the convergence of the power series requires a significant gap between the heaviest of the included states and the lightest of the not included states. For chiral perturbation theory the large gap emerges from the fact that one deals with Goldstone bosons. For the rest of the hadron spectrum the identification of a sizable gap is not so obvious. This issue will be picked up below.

For additional heavy degrees of freedom a power series in terms of the three-momenta of the heavy states and the four-momenta of the light states might be reasonable. Here the masses of the light states and the involved three-momenta of all states define the soft scale $p$. The mass(es) of the heavy states are part of the hard scale. This is the framework of baryon chiral perturbation theory [20]. Here it is crucial that the baryons cannot decay into mesons, in particular not into light mesons. Otherwise the scales are intertwined, because the three-momenta of the light decay products inherit the large scale of the decaying heavy state. This is one of the aspects which makes the inclusion of vector mesons so challenging (see below).

We have determined quantitatively what the phrase "an effective field theory works at low enough energies" means for strict mesonic $\chi$ PT. The lowest not considered degree of freedom is the sigma meson with a mass of about $600 \mathrm{MeV}$ [9]. This external hard scale seems to set the actual limit because it is lower than the intrinsic hard scale (2.7) set by the loops.

What was presented so far is the conservative picture. However, there might be some twists to the previous arguments: There are studies which suggest that the sigma meson is not a quarkantiquark state but rather a two-pion correlation (see, e.g., [25, 26] and references therein). If this is true, then there is no compelling reason to include the sigma as an explicit degree of freedom in an 
effective Lagrangian. An illustrative analogy might be the deuteron which emerges as a bound state from a calculation based on a Lagrangian which contains only nucleons (and mesons). In a similar way, the sigma could appear "dynamically" in the pion-pion phase shift. The Lagrangian might not contain the sigma meson. However, such a formalism can only work if the power counting is not directly applied to the observable quantities like the scattering amplitude. This would be like a truncated Born series applied to the problem of a bound state or a resonance. One rather has to sum up the whole Born series, i.e. really solve the Lippmann-Schwinger equation instead of a perturbative treatment. This translates to the demand to "unitarize" $\chi$ PT. The power counting should be applied to the determination of scattering kernels and not to scattering amplitudes. In other words, rescattering processes need to be resummed. From the puristic point of view this concept is unsatisfying because there are ambiguities in the unitarization/resummation procedure. On the other hand, unitarized versions of $\chi \mathrm{PT}$ have produced very promising results in the last few years $[27,25,26]$. In spite of the mentioned ambiguities the common feature of such approaches is the resummation of specific classes of diagrams (the two-particle reducible diagrams which correspond to rescattering). In such a framework the intrinsic hard scale needs to be re-evaluated. One needs to compare the size of two-particle irreducible loops and tree-level terms. Such an analysis has not been carried out yet. However, the successes of unitarized versions of $\chi$ PT up to energies of $1 \mathrm{GeV}$ and beyond suggest that the actual intrinsic hard scale is higher up than (2.7) once the two-particle reducible diagrams are properly resummed.

We have seen that it might be possible to circumvent both limitations of strict $\chi \mathrm{PT}$, the sigma mass and the scale (2.7). The next external hard scale is provided by the vector mesons. From all we know about QCD at present, it is highly unlikely that the vector mesons are two- or three-pion correlations. They are dominantly quark-antiquark states (see, e.g. [28] and references therein). Therefore, they need to be included as explicit degrees of freedom in an effective Lagrangian which is supposed to work in the region of low-lying mesonic resonances. If this is possible at all is a matter of active research. Further discussions and first steps towards this goal are presented in the next section. Before, however, the leading-order chiral Lagrangian for the sector of an odd number of pions will be briefly discussed.

In the sector of anomalous parity — where on the formal level a Levi-Civita tensor $\varepsilon_{\mu v \alpha \beta}$ is involved - the leading-order contribution to the effective field theory for Goldstone bosons is given by the Wess-Zumino-Witten action [29,30]. It is fully determined by the chiral anomaly and free of any undetermined low-energy parameters. It contributes at order $O\left(p^{4}\right)$. For the present purpose the only relevant part is the $\pi^{0} \gamma \gamma$ term given by

$$
\mathscr{L}_{\mathrm{WZW}}=\frac{3}{32 \pi^{2}} \frac{e^{2}}{f} \varepsilon_{\mu v \alpha \beta} F^{\mu v} F^{\alpha \beta} \operatorname{tr}\left(Q^{2} \Phi\right)
$$

with the electromagnetic field strength $F_{\mu \nu}=\partial_{\mu} A_{v}-\partial_{v} A_{\mu}$. This parameter-free Lagrangian provides an excellent description of the decay of the neutral pion into two real photons [20]. It will constitute one contribution to the pion transition form factor, see section 4 below.

\section{The vector-meson Lagrangian and its parameters}

In the last section the effective field theory for Goldstone bosons, $\chi \mathrm{PT}$, has been introduced. It has also been used to illustrate the general ideas of an effective field theory. For the formulation of 
an effective field theory for the energy region of vector mesons one has to consider the following questions: Should the vector mesons been introduced as light or heavy degrees of freedom? If light, which other mesons limit the applicability range, i.e. is there a large enough gap between the considered and not considered states? If light, where is the intrinsic hard scale? If heavy, how to deal with the fact that vector mesons can decay into light degrees of freedom (pions, photons)?

In the following, only preliminary answers can be provided which need to be further scrutinized in the future. However, one remarkable feature is that basically the same Lagrangian emerges (at the respective leading order), no matter whether one treats the vector mesons as light or as heavy degrees of freedom! This gives some credit to the proposed Lagrangian and might to some extent explain its phenomenological success even if a full-fledged effective field theory has not been established yet (or maybe even cannot be established).

The inclusion of vector mesons as light degrees of freedom has been proposed in [13] and further explored in $[14,15,26]$. Based on the hadrogenesis conjecture $[31,32,33,13]$ it has been argued that the other mesons in the energy range close to the vector mesons are dynamically generated from the coupled-channel interactions of pseudoscalar and vector mesons. Therefore, such states need not be considered explicitly in the effective Lagrangian. This implies that there is a sizable gap between the energy region of the lowest-lying vector and pseudoscalar mesons and the not considered other (quark-antiquark) states.

Whether the intrinsic hard scale is significantly larger than the vector-meson masses needs to be explored in loop calculations. It should be stressed again that exact unitarity, i.e. the resummation of two-particle reducible diagrams, is mandatory for an effective field theory which operates in the region of hadronic resonances. As already mentioned, the successes of unitarized versions of $\chi \mathrm{PT}$ suggest that the intrinsic hard scale could be significantly larger than $1 \mathrm{GeV}$. This would provide some room where an effective field theory of light vector mesons can be applicable.

The inclusion of vector mesons as strict heavy degrees of freedom has been pioneered in [34]. The focus has been set to reactions where a constant number of vector mesons remained throughout the whole respective process. In that way, the problem how to deal with decaying vector mesons has been avoided by hand. In the present work a more pragmatic way is suggested: Vector mesons are neither truly heavy, nor truly light. Their mass is close to the intrinsic hard scale (2.7) of (strict) $\chi$ PT. In that sense they are heavy. On the other hand, if vector mesons decay, the momenta of the decay products are still small compared to the scale (2.7). Slightly exaggerating, one might say that the vector-meson mass, $m_{V}$, is still heavy while $m_{V} / 2$ is already soft. This suggests to discuss separately the Lagrangian which includes the terms with one vector-meson field and the Lagrangian which contains the terms with two vector-meson fields. ${ }^{3}$ The first Lagrangian concerns the decays of vector mesons while the second Lagrangian concerns the propagation of vector mesons while absorbing or emitting light particles. Whether this pragmatic point of view is feasible, i.e. provides a systematic approach for loop calculations, needs to be seen in the future.

The leading-order Lagrangian collecting all terms with one vector-meson field is given by

$$
\mathscr{L}_{1}=-\frac{\mathrm{i}}{4} h_{P} m_{V} \operatorname{tr}\left(V_{\mu \nu}\left[U^{\mu}, U^{v}\right]\right)-\frac{1}{8} e_{V} m_{V} \operatorname{tr}\left(V^{\mu v} Q\right) F_{\mu \nu} .
$$

\footnotetext{
${ }^{3}$ More vector-meson fields are not needed at tree level for the processes of interest.
} 
The vector mesons are represented by anti-symmetric tensor fields, $V_{\mu \nu}[23,13]$. The phrase "leading order" has the following meaning: If one considers the vector mesons as semi-heavy in the sense that their mass is large (part of the hard scale) but the momenta of the decay products are already small $\sim p$, then one might count all derivatives (and the photon field encoded in the field strength $F_{\mu \nu}$ ) as soft in (3.1). The terms appearing in (3.1) are the only ones which have one vector-meson field and appear at order $O\left(p^{2}\right)$. Here, it is crucial that the vector mesons are represented by anti-symmetric tensor fields and not, e.g., by vector fields, $V_{\mu}$. In the latter case one would need more derivatives to construct the corresponding terms [24], e.g. one would have $\operatorname{tr}\left(\partial_{\mu} V_{v}\left[U^{\mu}, U^{v}\right]\right) \sim O\left(p^{3}\right)$ instead of the $h_{P}$ term in (3.1). It is important to stress that this statement applies to vector-meson fields which transform as ordinary matter fields with respect to chiral transformations. The treatment of vector mesons as gauge bosons [35] is not considered in the present work. The use of the anti-symmetric tensor representation is singled out by the fact that only here $O\left(p^{2}\right)$ terms are possible for structures with only one vector meson. In any other representation such structures would be at least $O\left(p^{3}\right)$.

If one considers the vector mesons as light degrees of freedom [13], the counting is the same, all derivatives are of order $p$. The complete Lagrangian is given in (3.1) and is of order $O\left(p^{2}\right)$.

The two parameters $h_{P}$ and $e_{V}$ can be determined from the decays $\rho \rightarrow 2 \pi$ and $\rho \rightarrow e^{+} e^{-}$[13]. The same results, but with higher accuracy, are obtained from a fit to the pion form factor [28].

For structures with two vector mesons the pertinent Lagrangian is

$$
\mathscr{L}_{2}=-\frac{1}{4} \operatorname{tr}\left(D_{\mu} V^{\mu \alpha} D^{v} V_{v \alpha}\right)+\frac{1}{8} m_{V}^{2} \operatorname{tr}\left(V_{\mu v} V^{\mu v}\right)+\frac{\mathrm{i}}{8} h_{A} \varepsilon_{\mu v \alpha \beta} \operatorname{tr}\left(\left\{V^{\mu v}, D_{\lambda} V^{\lambda \alpha}\right\} U^{\beta}\right) .
$$

Terms with more than one flavor trace have been neglected on account of the OZI rule [19, 23]. As a consequence the masses of rho and omega meson agree in this approach. Both are given by $m_{V}$.

If the vector mesons are considered as (semi-)heavy, then (3.2) is the complete leading-order $O(p)$ Lagrangian. It resembles the case of the nucleon $\chi \mathrm{PT}$ Lagrangian [20]. The $h_{A}$ interaction term translates to the well-known $g_{A}$ term of pion-nucleon interaction.

If the vector mesons are considered as light degrees of freedom [13], one counts all derivatives appearing in (3.2). The Lagrangian is of order $O\left(p^{2}\right)$. There are several more terms of this order. However, only one contributes to the processes of interest here. This term involves the square of the pion mass and is numerically rather unimportant $[13,15]$.

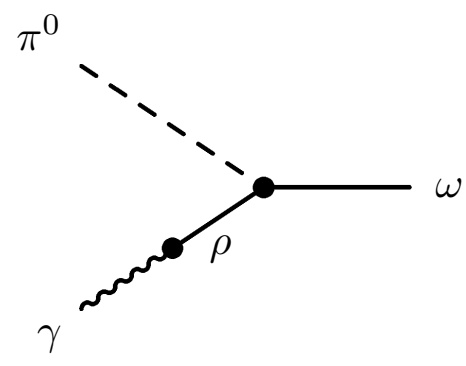

Figure 3: Two-step decay of an omega meson into a pion and a real photon.

The single free parameter $h_{A}$ can be fitted to the decay of an omega meson into a pion and a real photon. The corresponding diagram is shown in figure 3. Note that the process proceeds 
in two steps which involves the coupling constants $h_{A}$ for the $\omega$ to $\rho \pi$ decay and the constant $e_{V}$ from (3.1) for the direct coupling of the virtual $\rho$ to the photon. Since $e_{V}$ has been determined from another reaction $\left(\rho \rightarrow e^{+} e^{-}\right)$, the process $\omega \rightarrow \pi^{0}+\gamma$ can be used to fix $h_{A}$ [13, 15]. A direct interaction term for $\omega \rightarrow \pi^{0}+\gamma$ would involve more than two derivatives, i.e. it is of higher power than the terms of (3.1).

To summarize this section: No matter whether one treats vector mesons as semi-heavy or light degrees of freedom, one ends up with the Lagrangians presented in (3.1), (3.2). This is rather encouraging in view of the fact that the vector-meson mass is in between the hard and the soft scales of $\chi$ PT.

In the following, these Lagrangians are used together with the Lagrangians of $\chi \mathrm{PT}$ as given in (2.3) and (2.8). Their consequences are worked out at tree level or, if needed, including rescattering effects. Power counting issues will not be discussed anymore but deferred to future work.

\section{Electromagnetic form factors}

In the Lagrangians (3.1), (3.2) there are three free parameters. As already discussed they can be determined from two-body decays: $h_{p}$ from $\rho \rightarrow 2 \pi,\left|e_{V}\right|$ from $\rho \rightarrow e^{+} e^{-}$and $\left|h_{A} \cdot e_{V}\right|$ from $\omega \rightarrow \pi^{0} \gamma$. The numerical results are $[13,28,15]$

$$
\left|e_{V}\right| \approx 0.22, \quad h_{P} \approx 0.30, \quad\left|h_{A}\right| \approx 2.1 .
$$

There is a freedom to choose the sign of one coupling constant which multiplies an odd number of vector-mesons fields and the sign of one coupling constant which multiplies an odd number of pseudoscalar fields. This freedom has been used to specify the overall sign in the Wess-ZuminoWitten term (2.8) and the sign of the parameter $h_{P}$. To pin down the signs of the parameters $e_{V}$ and $h_{A}$ one needs more complex reactions. In other words: The sign of $h_{P}$ is pure convention, but the sign of $e_{V}$ relative to $h_{P}$ is a physics issue. The same applies to the sign of $h_{A}$ relative to the Wess-Zumino-Witten term (2.8).

Having fixed the parameter values (at least their absolute sizes), no further parameters are needed to determine

- the pion form factor, contained in the reaction $e^{+} e^{-} \rightarrow \pi^{+} \pi^{-}$,

- the omega transition form factor, $\omega \rightarrow \pi^{0} l^{+} l^{-}$,

- the single-virtual pion transition form factor, $\pi^{0} \rightarrow \gamma e^{+} e^{-}$,

- the double-virtual pion transition form factor, $\pi^{0} \rightarrow e^{+} e^{-} e^{+} e^{-}$.

It is important to note that at no stage any VMD assumption has entered. It might come out as a result of the calculations, but it is not put in by hand.

The process $e^{+} e^{-} \rightarrow \pi^{+} \pi^{-}$can take place via a direct coupling of the pions to the photon or via a vector meson. This is depicted in figure 4 . In the first case, the pions couple via their electric charge. This is described by the Lagrangian (2.3). In the second case the Lagrangian (3.1) provides the coupling of the virtual photon to the rho meson $\sim e_{V}$ and the coupling of the rho meson to the pions $\sim h_{P}$. 

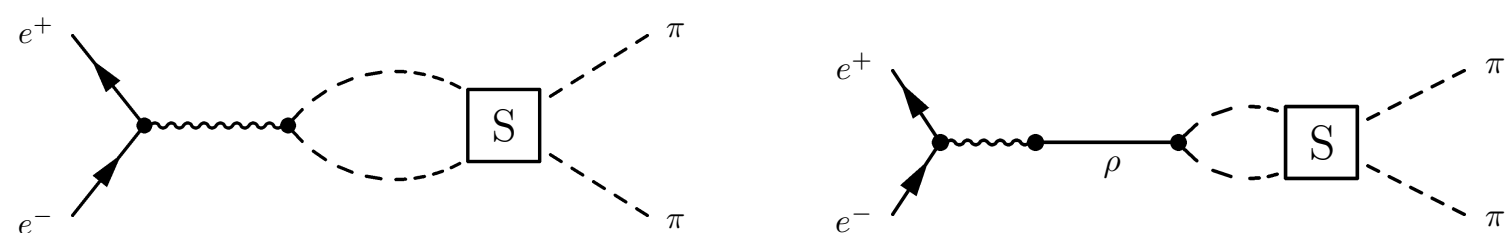

Figure 4: The two possible processes which lead from the initial state $e^{+} e^{-}$to the final state $\pi^{+} \pi^{-}$. The box denoted by "S" signals the rescattering of the pions. Figure taken from [28].
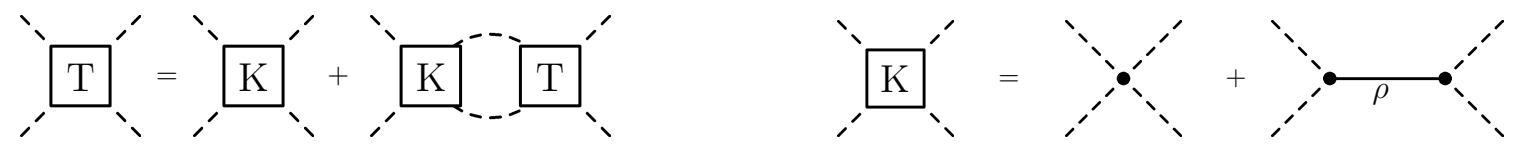

Figure 5: Bethe-Salpeter equation for the rescattering of pions in the $p$-wave channel. The $T$ matrix of pion-pion scattering is obtained from the scattering kernel denoted by $K$. Figure taken from [28].

A reliable description of the reaction $e^{+} e^{-} \rightarrow \pi^{+} \pi^{-}$and the pion form factor requires a resummation of the rescattering processes of the pions. In [28] this has been achieved by a Bethe-Salpeter equation. Schematically this is depicted in figure 5. The scattering kernel consists of two parts: The direct contact interaction between the pions is obtained from (2.3). The interaction between the pions and the rho meson comes again from the $h_{P}$ term of (3.1).

Concerning the comparison to VMD it is illuminating to discuss first the tree-level result for the pion form factor. Afterwards the full calculation including the rescattering process will be compared to the data.

The tree-level result is obtained from the diagrams of figure 4 by putting $S$ to 1, i.e. by dropping $S$ in the figure. It is common practice to normalize the pion form factor to the direct term (first term in figure 4 when putting $S$ to 1 ). In that way, the pion form factor becomes unity at the photon point. The tree-level pion form factor, as obtained from the Lagrangians (2.3) and (3.1) is given by

$$
F_{\pi}(s)=1+\frac{e_{V} h_{P} m_{V}^{2}}{16 e f^{2}} \frac{s}{m_{V}^{2}-s} .
$$

The invariant mass of the virtual photon is denoted by $s$. The VMD prediction is

$$
F_{\pi}^{\mathrm{VMD}}(s)=\frac{m_{V}^{2}}{m_{V}^{2}-s} .
$$

The two formulae would analytically agree for $e_{V} h_{P}=16 e f^{2} / m_{V}^{2} \approx 0.065$. With the values (4.1) one obtains $\left|e_{V}\right| h_{P} \approx 0.066$ which is obviously very close, provided one chooses a positive sign for $e_{V}$. Then a cancellation takes place: The two terms from the $\chi \mathrm{PT}$ Lagrangian (2.3) and the vector-meson Lagrangian (3.1), respectively, conspire such that the final result is close to VMD.

The full result based on the Lagrangians (2.3) and (3.1), but now including the rescattering of pions is shown in figure 6. Obviously one obtains an excellent result. Note that isospin breaking leads to $\rho-\omega$ mixing. This has not been included. Therefore, the sharp omega peak seen in the data cannot be reproduced.

The next quantity which will be considered is the electromagnetic form factor of the transition between an omega meson and a pion, see figure 7. The form factor is again normalized to the photon 


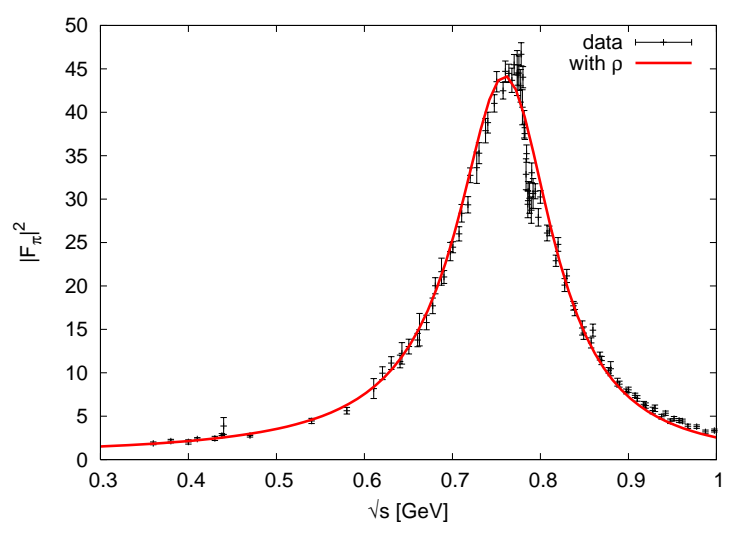

Figure 6: The pion form factor as compared to data [28].

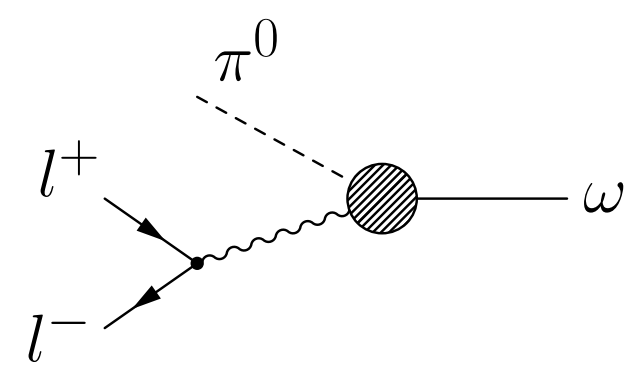

Figure 7: Generic picture for the omega-to-pion transition form factor. $l$ denotes a lepton.

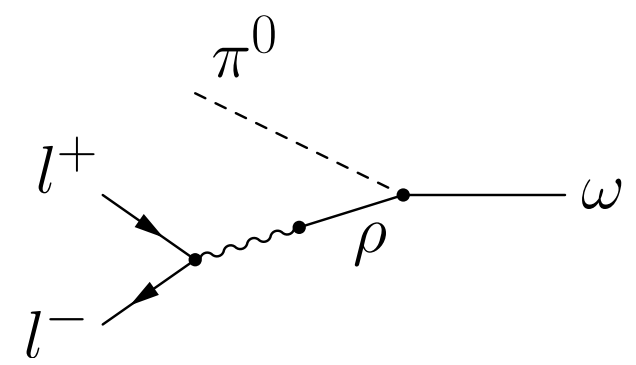

Figure 8: The omega-to-pion transition form factor in the present approach. The coupling $\rho \omega \pi$ is given by $h_{A}$ and the direct coupling of the rho meson to the photon by $e_{V}$.

point. Thus, it parametrizes the deviation from a structureless decay. In the present approach the process is described by the diagram shown in figure 8 . In principle, the intermediate rho meson could decay into two pions. Thus the rescattering of these pions which causes the width of the rho meson should be considered. It has been checked, however, that the decay process $\omega \rightarrow \pi^{0} l^{+} l^{-}$ with the lepton $l=e, \mu$ is not sensitive to the pion rescattering effects. Therefore, only the tree-level result will be presented in the following. The case would be different for the process $e^{+} e^{-} \rightarrow \omega \pi^{0}$ which probes the transition form factor at larger invariant masses. There, a proper description would require a coupled-channel calculation at least with the channels $\pi^{+} \pi^{-}$and $\omega \pi^{0}$, if not $K \bar{K}$. This is beyond the scope of the present work.

The process shown in figure 8 is proportional to the product $h_{A} \cdot e_{V}$. For the form factor, 


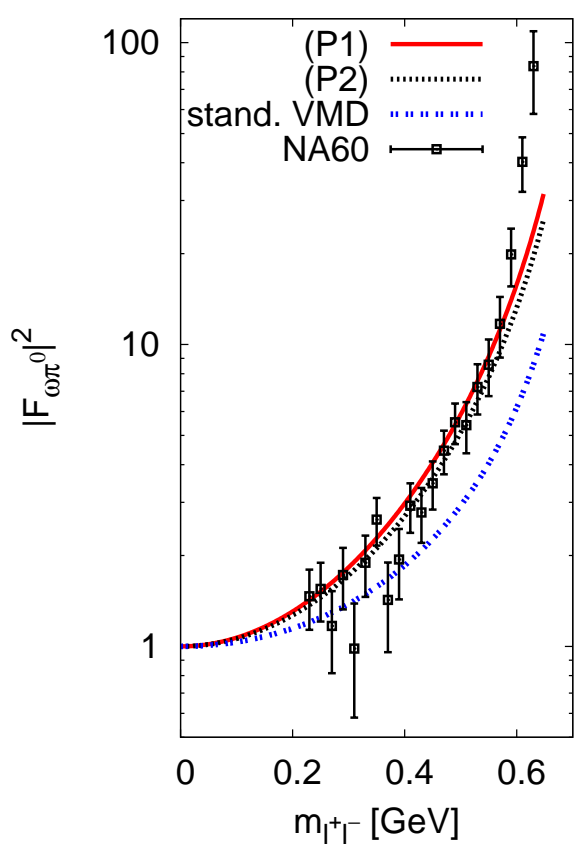

Figure 9: The omega to pion transition form factor. The present calculations are depicted by the full (red) line. Figure taken from [15].

however, this overall factor drops out because the form factor is normalized to the photon point. Therefore, the Lagrangians (3.1), (3.2) provide a parameter independent prediction for the omega transition form factor:

$$
F_{\omega \pi}(s)=\frac{m_{V}^{2}+s}{m_{V}^{2}-s}
$$

This deviates significantly from VMD:

$$
F_{\omega \pi}^{\mathrm{VMD}}(s)=\frac{m_{V}^{2}}{m_{V}^{2}-s} .
$$

Both form factors are compared to data from NA60 [8] in figure 9. Obviously, the NA60 data are much better described by the present approach (4.4) than by the VMD formula (4.5). A follow-up experiment using dielectrons instead of dimuons is planned by the WASA at COSY collaboration.

Next, the pion transition form factor is considered; at first, the single-virtual form factor which corresponds to the process $\pi^{0} \rightarrow \gamma e^{+} e^{-}$. It is depicted in figure 10. The present approach has two contributions, shown in figure 11, one from the Wess-Zumino-Witten term (2.8) and one from the vector-meson Lagrangians (3.1), (3.2). Note that the latter contribution vanishes if both photons are real, i.e. for $\pi^{0} \rightarrow 2 \gamma$. Thus, the excellent description of the latter process by the Wess-ZuminoWitten Lagrangian is not spoiled. The pion transition form factor, normalized to the photon point, 


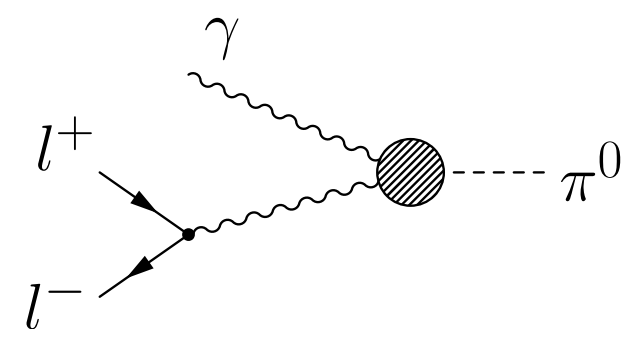

Figure 10: Generic picture for the pion-to-photon transition form factor. $l$ denotes a lepton.
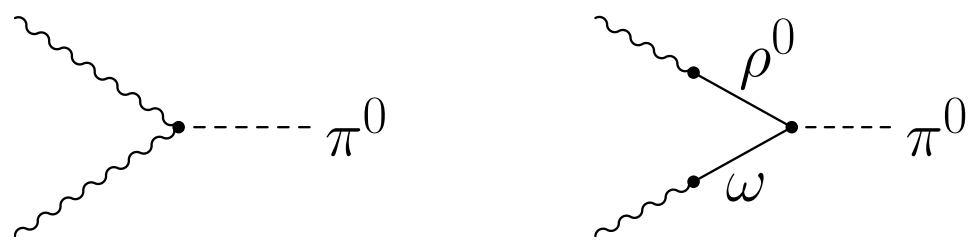

Figure 11: Left: The Wess-Zumino-Witten action contains a point interaction for one pion and two (real or virtual) photons (wavy lines). Right: The vector-meson Lagrangians (3.1), (3.2) induce an additional interaction between a pion and two photons.

i.e. normalized to the Wess-Zumino-Witten contribution, is given by

$$
F_{\pi \gamma}(s)=1+\frac{\pi^{2} h_{A} e_{V}^{2}}{12 e^{2}} \frac{s}{m_{V}^{2}-s} .
$$

The corresponding VMD formula is

$$
F_{\pi \gamma}^{\mathrm{VMD}}(s)=\frac{m_{V}^{2}}{m_{V}^{2}-s}
$$

Note that the masses of rho and omega meson are not distinguished here. Of course, there are kinematical situations where the tree-level results are not enough. For the comparison of the present approach to the VMD result, however, it is most transparent to look at the tree-level formulae.

The formulae (4.6) and (4.7) would agree analytically for $h_{A} e_{V}^{2}=12 e^{2} / \pi^{2} \approx 0.11$. Using the numerical values from (4.1) one obtains $\left|h_{A}\right| e_{V}^{2} \approx 0.10$. Thus, the formula (4.6) is again numerically close to the VMD result, provided one uses a positive sign for $h_{A}$. Another cancellation has taken place, now between the Wess-Zumino-Witten term and the vector-meson contribution. As already mentioned in the introduction, the VMD prediction is close to the experimental result. Therefore, the present approach agrees also with the experimental result for the single-virtual pion transition form factor.

However, the picture changes for the double-virtual transition form factor: It is given by

$$
\begin{aligned}
F\left(s_{1}, s_{2}\right) & =1+\frac{\pi^{2} h_{A} e_{V}^{2}}{12 e^{2}} \frac{m_{V}^{2}\left(s_{1}+s_{2}\right)}{\left(m_{V}^{2}-s_{1}\right)\left(m_{V}^{2}-s_{2}\right)} \\
& \approx 1+\frac{m_{V}^{2}\left(s_{1}+s_{2}\right)}{\left(m_{V}^{2}-s_{1}\right)\left(m_{V}^{2}-s_{2}\right)} \\
& =1 \underbrace{-\frac{m_{V}^{2}}{m_{V}^{2}-s_{1}}-\frac{m_{V}^{2}}{m_{V}^{2}-s_{2}}}_{\text {"half" VMD }}+2 \underbrace{\frac{m_{V}^{4}}{\left(m_{V}^{2}-s_{1}\right)\left(m_{V}^{2}-s_{2}\right)}}_{\text {VMD type }}
\end{aligned}
$$


while the VMD formula is simply

$$
F_{\mathrm{VMD}}\left(s_{1}, s_{2}\right)=\frac{m_{V}^{4}}{\left(m_{V}^{2}-s_{1}\right)\left(m_{V}^{2}-s_{2}\right)} .
$$

The two virtualities of the photons are denoted by $s_{1}$ and $s_{2}$. Obviously, (4.8) is different from VMD for $s_{1}, s_{2} \neq 0$. Future experimental data for the double-virtual transition form factor are crucial to distinguish between the present approach and the VMD scenario. As already mentioned in the introduction such data are also an important input for the calculation of the hadronic contribution to the gyromagnetic ratio of the muon.

\section{Further discussion, summary and outlook}

The respective lowest-order Lagrangians of chiral perturbation theory for the sectors with an even and with an odd number of pions have been combined with a Lagrangian for vector mesons as given in (3.1) and (3.2). Also these ingredients have been motivated by effective field theory ideas and different options and open problems concerning the proper power counting in the energy region of vector mesons have been discussed. Though several issues are unsettled, it is appealing that the constructed vector-meson Lagrangian can be motivated in two different way, treating the vector mesons as light or as (semi-)heavy degrees of freedom.

The developed formalism can be used to calculate the following quantities:

- All three parameters of the vector-meson Lagrangian are fitted to the two-body decays $\rho \rightarrow$ $2 \pi, \rho / \omega \rightarrow l^{+} l^{-}(l=$ lepton $)$ and $\omega \rightarrow \pi^{0} \gamma[13]$.

- The reaction $e^{+} e^{-} \rightarrow \pi^{+} \pi^{-}$and the corresponding pion form factor is in good agreement with the data. Intimately connected to this is also a good description of the $p$-wave pion-pion scattering phase shift (not shown here) [28].

- The decay $\omega \rightarrow \pi^{0} l^{+} l^{-}$and the corresponding omega transition form factor is much better described than with the vector-meson dominance (VMD) model [15].

- The single-virtual pion transition form factor, as, e.g., measured in the decay $\pi^{0} \rightarrow \gamma e^{+} e^{-}$, turns out to be close to the VMD result and therefore also close to the data.

- A prediction is provided for the double-virtual pion transition form factor which disagrees with the VMD prediction.

- The decay rate of $\omega \rightarrow \pi^{+} \pi^{-} \pi^{0}$ is reproduced very well (not shown here) [14].

- The reaction $e^{+} e^{-} \rightarrow \pi^{+} \pi^{-} \pi^{0}$ is presently investigated [36]. Like for the pion transition form factor there is an interplay between the Wess-Zumino-Witten term and the vector-meson contribution.

It is worth to summarize the comparison to the VMD model: Where the experimental data are well described by VMD (pion form factor and single-virtual pion transition form factor), the present approach contains two terms, one from chiral perturbation theory and one including vector 
mesons. These two terms conspire such that cancellations occur which bring the results very close to the VMD results. Note that this has not been adjusted by hand. The coupling constants of the vector-meson Lagrangian can been determined independently using the two-body decays, not the form factors. For the pion form factor the mentioned cancellation might be related to the KSFR relation [24]. On the other hand, the other cancellation which takes place for the (single-virtual) pion transition form factor has nothing to do with KSFR. In addition, the KSFR relation does not follow from chiral symmetry alone. On way to derive it is to use in addition the high-energy behavior of the pion form factor [24]. It is not clear whether high-energy constraints should be applied to a low-energy theory. Obviously, the observed cancellations are very interesting, but their microscopic reason remains an open issue at present.

In any case it is appealing to have a formalism which is flexible enough to reproduce VMD results but can also go beyond it. Indeed, for the omega transition form factor the VMD prediction drastically deviates from the data. Here, the present approach provides only one contribution, not two which could potentially show a cancellation effect. Qualitatively resembling the VMD model, the present approach provides a parameter independent prediction for the omega transition form factor. However, it is quantitatively different from VMD, namely $\left(m_{V}^{2}+s\right) /\left(m_{V}^{2}-s\right)$ instead of $m_{V}^{2} /\left(m_{V}^{2}-s\right)$. The former expression provides a much better description of the experimental data.

A challenge for the future is to establish a systematic power counting for the energy region of vector mesons. Here calculations beyond leading order, including in particular loop calculations, are an important issue. A second line of development is the extension to three flavors with the inclusion of the $\eta^{\prime}$. Finally, a better (microscopic?) understanding of the observed cancellations would be desirable to further appreciate the successes of VMD and to see its limitations.

Acknowledgments: S.L. thanks M.F.M. Lutz for collaboration and discussions. He also acknowledges stimulating discussions with A. Kupsc. Finally he wants to thank the organizers of Bormio 2012 for their excellent work.

\section{References}

[1] H. W. Kendall, Deep inelastic scattering: Experiments on the proton and the observation of scaling, Rev. Mod. Phys. 63 (1991) 597 (Nobel prize lecture 1990).

[2] S. Leupold, V. Metag and U. Mosel, Hadrons in strongly interacting matter, Int. J. Mod. Phys. E 19 (2010) 147 [arXiv:0907.2388 [nucl-th]].

[3] B. Friman et al., The CBM physics book: Compressed baryonic matter in laboratory experiments, Lect. Notes Phys. 814 (2011) 1.

[4] F. Jegerlehner and A. Nyffeler, The muon g-2, Phys. Rept. 477 (2009) 1 [arXiv:0902.3360 [hep-ph]].

[5] J. J. Sakurai, Currents and mesons, University of Chicago Press, Chicago, USA, 1969.

[6] F. Klingl, N. Kaiser and W. Weise, Effective Lagrangian approach to vector mesons, their structure and decays, Z. Phys. A 356 (1996) 193 [arXiv:hep-ph/9607431].

[7] L. G. Landsberg, Electromagnetic decays of light mesons, Phys. Rept. 128 (1985) 301.

[8] R. Arnaldi et al. [NA60 Collaboration], Study of the electromagnetic transition form-factors in $\eta \rightarrow \mu^{+} \mu^{-} \gamma$ and $\omega \rightarrow \mu^{+} \mu^{-} \pi^{0}$ decays with NA60, Phys. Lett. B 677 (2009) 260 [arXiv:0902.2547 [hep-ph]]. 
[9] K. Nakamura et al. [Particle Data Group], Review of particle physics, J. Phys. G 37 (2010) 075021.

[10] B. Borasoy and R. Nissler, Two photon decays of $\pi^{0}, \eta$ and $\eta^{\prime}$, Eur. Phys. J. A 19 (2004) 367 [hep-ph/0309011].

[11] T. Petri, Anomalous decays of pseudoscalar mesons, arXiv:1010.2378 [nucl-th].

[12] C. Terschlüsen, Electromagnetic transition form factors of pseudoscalar and vector mesons, Physics Diploma Thesis, Giessen University, 2010.

[13] M. F. M. Lutz and S. Leupold, On the radiative decays of light vector and axial-vector mesons, Nucl. Phys. A 813 (2008) 96 [arXiv:0801.3821 [nucl-th]].

[14] S. Leupold and M. F. M. Lutz, Hadronic three-body decays of light vector mesons, Eur. Phys. J. A 39 (2009) 205 [arXiv:0807.4686 [hep-ph]].

[15] C. Terschlüsen and S. Leupold, Electromagnetic transition form factors of light vector mesons, Phys. Lett. B 691 (2010) 191 [arXiv:1003.1030 [hep-ph]].

[16] C. Terschlüsen, S. Leupold and M.F.M. Lutz, in preparation.

[17] S. Weinberg, Phenomenological Lagrangians, Physica A 96 (1979) 327.

[18] J. Gasser and H. Leutwyler, Chiral perturbation theory to one loop, Annals Phys. 158 (1984) 142.

[19] J. Gasser and H. Leutwyler, Chiral perturbation theory: Expansions in the mass of the strange quark, Nucl. Phys. B 250 (1985) 465.

[20] S. Scherer, Introduction to chiral perturbation theory, Adv. Nucl. Phys. 27 (2003) 277, [arXiv:hep-ph/0210398].

[21] J. Bijnens, G. Colangelo and G. Ecker, The mesonic chiral Lagrangian of order $p^{6}$, JHEP 9902 (1999) 020 [hep-ph/9902437].

[22] T. Ebertshäuser, H. W. Fearing and S. Scherer, The anomalous chiral perturbation theory meson Lagrangian to order $p^{6}$ revisited, Phys. Rev. D 65 (2002) 054033 [hep-ph/0110261].

[23] G. Ecker, J. Gasser, A. Pich and E. de Rafael, The role of resonances in chiral perturbation theory, Nucl. Phys. B 321 (1989) 311.

[24] G. Ecker, J. Gasser, H. Leutwyler, A. Pich and E. de Rafael, Chiral Lagrangians for massive spin 1 fields, Phys. Lett. B 223 (1989) 425.

[25] J. A. Oller and E. Oset, N/D description of two meson amplitudes and chiral symmetry, Phys. Rev. $D$ 60 (1999) 074023 [hep-ph/9809337].

[26] I. V. Danilkin, L. I. R. Gil and M. F. M. Lutz, Dynamical light vector mesons in low-energy scattering of Goldstone bosons, Phys. Lett. B 703 (2011) 504 [arXiv:1106.2230 [hep-ph]].

[27] A. Gomez Nicola and J. R. Pelaez, Meson meson scattering within one loop chiral perturbation theory and its unitarization, Phys. Rev. D 65 (2002) 054009 [hep-ph/0109056].

[28] S. Leupold, Information on the structure of the rho meson from the pion form-factor, Phys. Rev. D 80 (2009) 114012 [Erratum-ibid. D 83, 079902 (2011)] [arXiv:0907.0100 [hep-ph]].

[29] J. Wess and B. Zumino, Consequences of anomalous Ward identities, Phys. Lett. B 37 (1971) 95.

[30] E. Witten, Global aspects of current algebra, Nucl. Phys. B 223 (1983) 422.

[31] M. F. M. Lutz and E. E. Kolomeitsev, Relativistic chiral SU(3) symmetry, large N(c) sum rules and meson baryon scattering, Nucl. Phys. A 700 (2002) 193 [arXiv:nucl-th/0105042]. 
[32] M. F. M. Lutz, G. Wolf and B. Friman, Scattering of vector mesons off nucleons, Nucl. Phys. A 706, 431 (2002) [Erratum-ibid. A 765, 431 (2006)] [arXiv:nucl-th/0112052].

[33] M. F. M. Lutz and E. E. Kolomeitsev, On meson resonances and chiral symmetry, Nucl. Phys. A 730 (2004) 392 [arXiv:nucl-th/0307039].

[34] E. E. Jenkins, A. V. Manohar and M. B. Wise, Chiral perturbation theory for vector mesons, Phys. Rev. Lett. 75 (1995) 2272 [hep-ph/9506356].

[35] M. Harada and K. Yamawaki, Hidden local symmetry at loop: A New perspective of composite gauge boson and chiral phase transition, Phys. Rept. 381 (2003) 1 [hep-ph/0302103].

[36] B. Strandberg, Physics Master Thesis, Uppsala University, in preparation. 